\title{
X-ray Synchrotron Polarization from Turbulent Plasmas in Supernova Remnants
}

\author{
Matthew G. Baring \\ Department of Physics and Astronomy - MS 108, Rice University, \\ 6100 Main Street, Houston, Texas 77251-1892, USA \\ email: baring@rice.edu
}

\begin{abstract}
As supernova remnants (SNRs) age, they become efficient cosmic ray accelerators at their outer shell shocks. The current paradigm for shock acceleration theory favors turbulent field environs in the proximity of these shocks, turbulence driven by current instabilities involving energetic ions. With the imminent prospect of dedicated X-ray polarimeters becoming a reality, the possibility looms of probing turbulence on scales that couple to the super- $\mathrm{TeV}$ electrons that emit X-rays. This paper presents model X-ray polarization signatures from energetic electrons moving in simulated MHD turbulence of varying levels of "chaos." The emission volumes are finite slabs that represent the active regions of young SNR shells. We find that the turbulent field energy must be quite limited relative to that of the total field in order for the X-ray polarization degree to be as strong as the radio measures obtained in some remnants. Results presented are pertinent to the planned IXPE and XIPE polarimeters.
\end{abstract}

Keywords. supernova remnants, cosmic rays, radiation mechanisms: nonthermal, polarization, plasmas, turbulence.

\section{Introduction}

One of the most important contributions of the Chandra X-ray Observatory to supernova remnant (SNR) science has been the discovery of thin synchrotron rims in the outer shells of several remnants. This advance was enabled by Chandra's exquisite imaging capability. Among these sources are SN1006 (Long et al. 2003) and Cassiopeia A (Vink \& Laming 2003), the original first light target for the Observatory. The sharp rise and fall of the X-ray flux in these filamentary rims, on angular scales of a few arcseconds, implies a very short synchrotron cooling timescale. This yields the unavoidable interpretation that the embedded magnetic field is on the order of 20-70 $\mu$ Gauss, substantially above values normally interpreted from standard invocations of field compression in MHD shocks. Thus a quandary emerged: how could such anomalous field enhancements come about?

In the years just prior to and around this time, the concept of turbulent amplification of magnetic fields mediated by cosmic ray-driven instabilities in SNR shocks was being developed, led by the papers by Lucek \& Bell (2000) and Bell (2005). The anisotropy of the most energetic ions on the scales of the thickness of remnant shells seeds growth of MHD fluctuations to the levels of $(\delta B / B)^{2} \sim \mathcal{M}_{\mathrm{A}} P_{\mathrm{cr}} / \rho u^{2} \gtrsim 3-10$. Here $\mathcal{M}_{\mathrm{A}}$ is the Alfvénic Mach number, $P_{\mathrm{cr}}$ is the cosmic ray $(\mathrm{CR})$ pressure, and $\rho u^{2}$ is the fluid ram pressure. Basically, the CR pressure gradient does work on the field energy density. The interplay between the thermal gas, the cosmic rays and the field energy introduces inherently non-linear elements to the dynamics of the shock environs (e.g. Vladimirov, Ellison \& Bykov 2006). The observation of thin rims propelled the extensive development of models of the so-called Bell instability, and the paradigm that SNR shells contain fields that are turbulent and enhanced has become very popular. 
We are now at the dawn of a new era in X-ray imaging, namely with the advent of X-ray polarimetry. NASA announced earlier this year the selection of the Imaging X-ray Polarimetry Explorer (IXPE, see Weisskopf et al. 2013) as a Small Explorer mission, to be launched in four or so years. This will serve as a pathfinder for discovery of polarized sources in X-rays, with a special focus on imaging and therefore on supernova remnants. IXPE's nominal 30" angular resolution will be able to probe the diffusive scales of the most energetic electrons emitting synchrotron X-rays in SNRs. It will thus be able to cast light on the level and nature of turbulence in SNR rims and shells, with around 8 prime SNR targets in its advertised science program (Weisskopf et al. 2013). $\dagger$ Subsequent Xray polarimetry initiatives such as XIPE (Soffitta et al. 2013) and eXTP offer additional prospects for probing the SNR environment in the not too distant future.

In preparation for such a data influx, exploration of what one might expect to see with an X-ray polarimeter is strongly motivated. A seminal study of the flux and polarization degree variations in turbulent field models of synchrotron-emitting remnants was published in Bykov et al. (2009), outlining how images sampling various angular scales can capture the information of field turbulence. Here we present results from an incipient simulation of synchrotron radiation from charges in prescribed MHD turbulence using a somewhat different construction, but notably with a decidedly different emphasis. Here the simulation output provides measures of the fluctuations, mean and standard deviation of the polarization Stokes parameters, and demonstrates the strong correlation between these and the variance of Kolmogorov turbulence in SNR shells.

\section{Synchrotron Polarization from Electrons in Magnetic Turbulence}

To assess the nature of polarized synchrotron emission in turbulence near SNR shocks, a simulation of charge motion in prescribed magnetostatic field fluctuations was developed. The construction was similar to the approach of Giacalone \& Jokipii (1999) in their charge diffusion study, in that 1D turbulence was superposed upon a uniform background field $\mathbf{B}_{0}$, which is radially-directed along the $x$-axis. The MHD turbulence assumes the form

$$
\delta \mathbf{B}=\sum_{k} \delta B_{k}\left\{\cos \phi_{\perp} \hat{y}+\sin \phi_{\perp} \hat{z}\right\} \cos \left(k x+\phi_{k}\right) .
$$

The slab nature is embodied in the property that the wavenumbers $\mathbf{k}$ of turbulence only possess a component $k \hat{x}$ along the direction of the unperturbed field $\mathbf{B}_{0}$, and the transverse field perturbations are in the $(y, z)$-plane, perpendicular to the plane of the sky (adopted for simplicity though not necessarily true, it is a testable assumption nonetheless). The wave phase $\phi_{k}$ and the azimuthal orientation $\phi_{\perp}$ were chosen randomly. The power spectrum of the fluctuations was presumed to have a 1D Kolmogorov form:

$$
\frac{\left\langle\delta B_{k}\right\rangle^{2}}{B_{0}^{2}}=\sigma^{2}\left(\frac{k}{k_{-}}\right)^{-5 / 3} \quad, \quad k_{-} \leqslant k \leqslant k_{+} .
$$

Here $\sigma^{2}$ is the wave variance at the base of the inertial range, i.e. at $k=k_{-} \equiv 2 \pi / \lambda_{\text {stir }}$, with $\lambda_{\text {stir }} \sim R_{\text {shell }} / 3$ being the stirring scale for the cascading turbulence. Also, for the simulations herein, $k_{+}=100 k_{-}$was adopted with values of $k$ sampled randomly on a logarithmic scale over the inertial range. Representative simulated turbulent fields are illustrated in Fig. 1 via their projections onto the sky plane, which is taken to encapsulate the radial vector through the slab. The absence of any time dependence (magnetostatic assumption) in $\delta \mathbf{B}$ is a tantamount to presuming non-relativistic plasma dynamics.

$\dagger$ See also the IXPE web page at https://wwwastro.msfc.nasa.gov/ixpe/. 

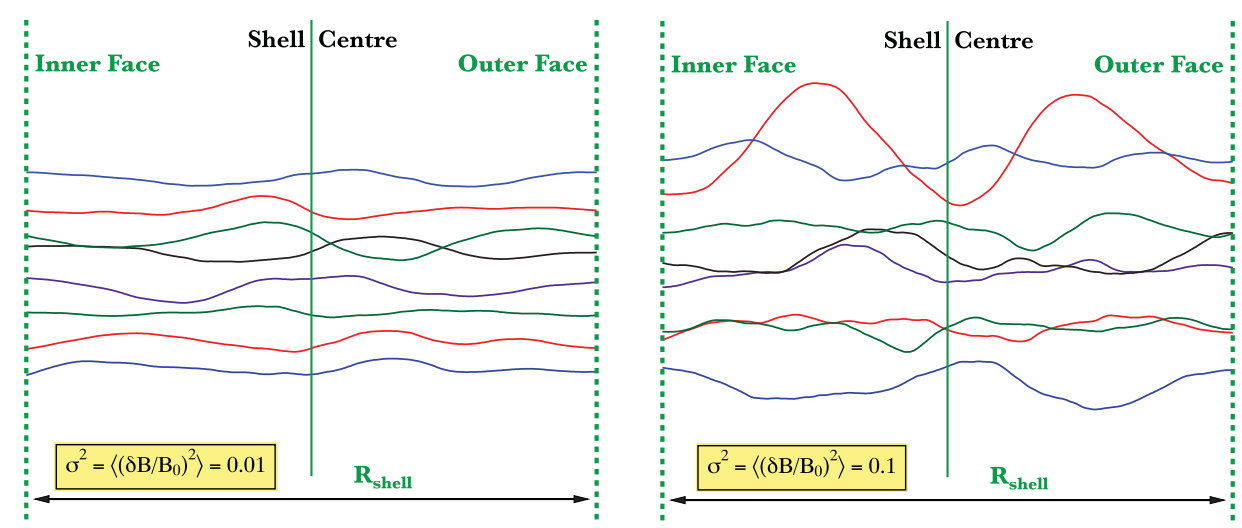

Figure 1. Schematics for the geometry of SNR shell containing turbulent fields. The depicted field lines are from the prescription of Kolmogorov slab turbulence in Eq. (2.2), with the variance $\sigma^{2}=\left\langle\left(\delta B / B_{0}\right)^{2}\right\rangle$ of the turbulence at the stirring scale $R_{\text {shell }} / 3$ being $\sigma^{2}=10^{-2}$ for the left panel, and $\sigma^{2}=10^{-1}$ for the right panel. Only the projections of the field lines in the plane of the Figure are represented; the color coding serves only to aid distinguishability of the lines.

Yet the synchrotron-radiating electrons are indeed ultra-relativistic. They were injected at the inner boundary $(x=-10)$ of the slab, distributed randomly in the $(y, z)$ plane, and with the initial momentum vector directions selected randomly from an isotropic distribution. The Lorentz factors were selected randomly on a logarithmic scale from a power law distribution over a narrow range spanning a factor of two in $\Gamma_{e}$. The actual scale was a convolution of $\Gamma_{e} / \sqrt{B_{0}}$ so as to position the synchrotron spectral information neatly in the X-ray band. The electrons were then propagated into the slab, solving the Lorentz force equation in the turbulent field structure in incremental intervals along their trajectories using a fourth-order Euler ODE solver. Their paths and acceleration vectors were logged for use in the polarization algorithms.

\subsection{Synchrotron Polarization Characteristics}

To develop a general idea of the properties of synchrotron radiation polarization in SNR shells, the synchrotron emissivity is integrated over the charges' trajectories. This polarized emissivity is principally dependent on three quantities at each point along the lepton path: the magnetic field vector and the electron's Lorentz factor and pitch angle. Since the charges were injected into the slab with an isotropic angular distribution, provided the turbulence is not too large, they retain approximate isotropy throughout the slab; this is relinquished somewhat when $\delta B / B \sim 1$. The local acceleration vector determines the instantaneous pitch angle, and combined with $\mathbf{B}$, they serve as parametric input for the standard synchrotron emissivity formulae (e.g. see Rybicki \& Lightman 1979). The electron Lorentz factors are distributed, and for our purposes here, a power-law distribution $n_{e}(\gamma) \propto \gamma^{-p}$ with $p=3$ was presumed. This generates a synchrotron intensity spectrum $I_{\nu} \propto \nu^{-1}$, which approximates the steep synchrotron slopes in the keV X-ray band seen in SNRs that are interpreted as portions of exponential turnovers. Accordingly, the assignment of synchroton photon electric field vectors can be made with the usual \| and $\perp$ designation and textbook probabilities. Thus

$$
\Pi \equiv \frac{I_{\perp}-I_{\|}}{I_{\perp}+I_{\|}} \rightarrow \frac{p+1}{p+7 / 3} \rightarrow \frac{3}{4}
$$

is the degree of polarization for a uniform, unturbulent field $(\sigma \rightarrow 0)$. 
These polarization assignments are then converted into contributions to the Stokes parameters as an electron continues its trajectory. If the projection of the magnetic field onto the sky plane $(x, y)$ lies parallel to the mean field vector $\mathbf{B}_{0}$ in the $x$-direction, then one is at liberty to assign $Q=I, U=0$ for the $\|$ state, and $Q=-I, U=0$ for the $\perp$ state. Since this field orientation does not point to an observer, the zero circular polarization sets $V=0$. Adding components to $\mathbf{B}$ in the $z$-direction perpendicular to the sky plane does not alter this polarization, but it does change the synchrotron intensity, since the field vector has been modified. Adding a field component in the $y$-direction amounts to rotating the sky plane-projected field about the $z$-axis through some angle $\chi$ (the position angle), and this then mixes the Stokes parameters (tensor elements) so that $Q=I \cos 2 \chi, U=I \sin 2 \chi$ for a $\|$ state contribution, and $Q=-I \cos 2 \chi, U=$ $I \sin 2 \chi$ for a $\perp$ state one. These are then added for each step along the electron path, sampling different local field vectors and varying pitch angles. As long as the local field components are inferior to the total sky plane-projected $\mathbf{B}$, the circularity is simply $V=0$. The result is an integrated ensemble of Stokes $Q, U$ and $I$ information.

For the purposes of illustration, the emission was discretized using the cubic slab structure in a sequence of slices abutting each other along the shock face, but also in bins of equal thickness in the shock normal $(x)$ direction. Thus, a slice constitutes a volume $-r \leqslant x \leqslant+r,-n r / N \leqslant y \leqslant+n r / N,-r \leqslant z \leqslant+r$, and each bin defines a rectangular prismatic rod of volume $-n r / N \leqslant x \leqslant+n r / N,-n r / N \leqslant y \leqslant+n r / N,-r \leqslant z \leqslant+r$ (for integer $n \leqslant N$ ) that is aligned normal to the sky plane. Here $r$ is the slab halfthickness, which scales with the thickness $R_{\text {shell }}$ of the SNR shell; $r=10$ is chosen here. Also, $N=4$ is chosen for the purposes of illustration. This approximates the sky plane pixelation density achievable with IXPE for typical remnants as afforded by its angular resolution of $\sim 30^{\prime \prime}$ - see various figures in Bykov et al. (2009) for a range of intensity imagery realizable with X-ray polarimetry observations of turbulent SNR shells.

The synchrotron Stokes parameter contributions from $10^{4}$ electrons injected with a narrow range of Lorentz factors into slab at the $x=-10$ boundary were recorded, added, and assigned to each $(x, y)$ pixel according to the position of each charge as it moved along its trajectory. Results for two turbulence cases are ilustrated in Figs. 2 (for $\sigma^{2}=10^{-2}$ ) and 3 (for $\sigma^{2}=0.1$ ). The four panels each plot histograms for each slice, color-coded to enhance the visual distinguishability, given the overlapping/entanglement of the histograms. The intermingling of such traces is expected because of the diffusive meandering of electrons relative to pure gyrohelixes in uniform magnetic fields. The benchmark values for a uniform background field, $\mathbf{B}_{0}$ are indicated with the horizontal red lines with double arrowheads. For the chosen index, the polarization degree $\Pi=\sqrt{Q^{2}+U^{2}} / I$ realizes a value of $3 / 4$, and the position angle is $\chi=0$ for the Stokes parameter convention adopted here. The introduction of turbulence reduces the polarization degree value as expected, with $\Pi(\sigma) \simeq 3 / 4-3 \sigma^{2} / 2$. The contributions of $Q$ and $U$ to the deviation from the uniform field value are of the same magnitude, but not identical. While $Q$ fluctuates a little in departing from the baseline $3 / 4$ value, the statistical scatter in values of $U$ is an unambiguous marker of the turbulence. The average value of $U$ is close to zero, but the standard deviation scales as $\sigma^{2}$. Accordingly, collecting information about the mean and standard deviation of the position angle $\chi$ affords a second direct diagnostic on the strength of the turbulence, namely $|\chi| \sim \sigma^{2}$. This latter result is anticipated: the variance in polarization direction should directly scale with the variance in magnetic field directions. These results obviously are obtained in the domain when the pixel scale is of the order of the turbulence stirring scale. When the stirring scale is much smaller than the polarimeter resolution scale, the laminar structure of the field will emerge in the results, corresponding to $\sigma^{2} \ll 1$ domains. 

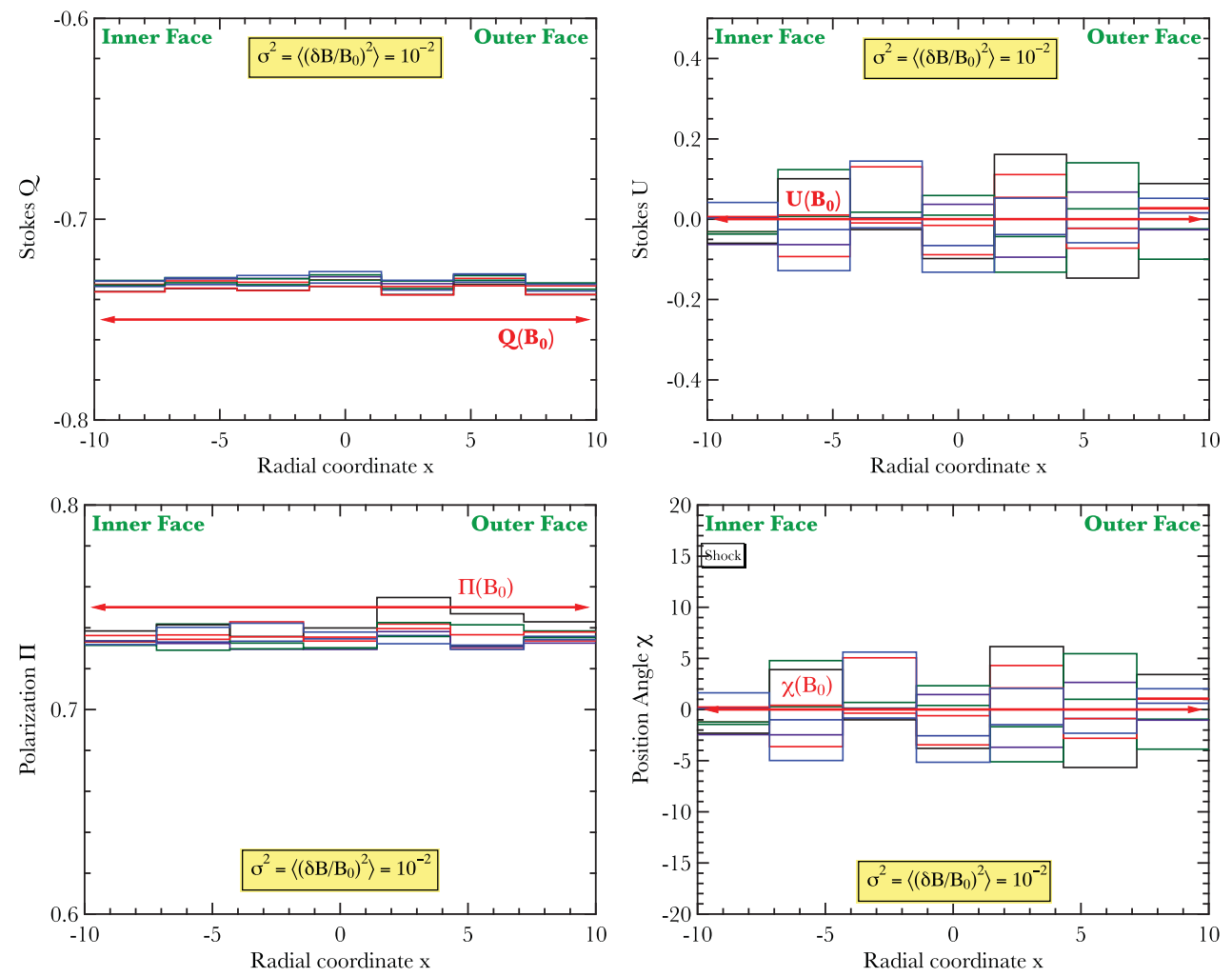

Figure 2. Synchrotron X-ray polarization quantities from power-law electrons $n_{e}(\gamma) \propto \gamma^{-p}$ with distribution index $p=3$, for a sequence of slices of the shell that are oriented in the $x$-direction normal to the planar shock, and whose centers are laterally distributed with equidistant separations along the shock. Each section of a histogram thus represents the polarization measure associated with a rectangular prism segment of a slice, with a square cross section in the plane of Fig. 1. The color coding is to aid distinguishability of results for different slices, with color correspondence through all panels. The field turbulence had a Kolmogorov spectrum above the stirring scale $\Delta x=20 / 3$, at which the variance was $\sigma^{2}=10^{-2}$. The Stokes $\mathrm{Q}$ and $\mathrm{U}$ values are depicted in the upper panels, with circularity $V$ being zero. The polarization degree $\Pi$ and position angle $\chi=1 / 2 \arctan (U / Q)$ are in the bottom two panels. The values of all four quantities for a uniform field $\mathbf{B}_{0}$ are indicated by the red double-headed long arrows.

These illustrations provide key insights into the coupling between field turbulence and X-ray synchrotron polarization, even though the slab geometry does not precisely model the spatial non-uniformity and curvature of SNR shell environments. The polarization degrees represented in the two Figures are substantially higher than those measured in the radio for SN 1006 in Reynolds \& Gilmore (1993) and Reynoso, Hughes \& Moffett (2013), suggesting that the variance of field turbulence on the diffusion/gyrational scales of $\mathrm{GeV}$ radio-emitting electrons (much smaller than $R_{\text {shell }}$ ) must be on the order of $\sigma^{2} \lesssim 1$. Yet, the position angle imagery for the VLA data on SN 1006 indicates a coherent component to the field. The X-ray band samples much larger diffusion scales, on the order of the shell thickness. On these lengthscales, the cosmic-ray driven magnetic field amplification models predict $\delta B / B \sim 1$, for which the results here suggest not only polarization degrees $\Pi<0.4$, but that the position angle information in the $2-8 \mathrm{keV}$ window should be highly disordered, with the variance in $\chi$ providing a direct measure of the variance in the field fluctuations. Moreover, the coupling constant should be dependent on the binning or pixelation size of the data, providing constraints on the stirring scale for 

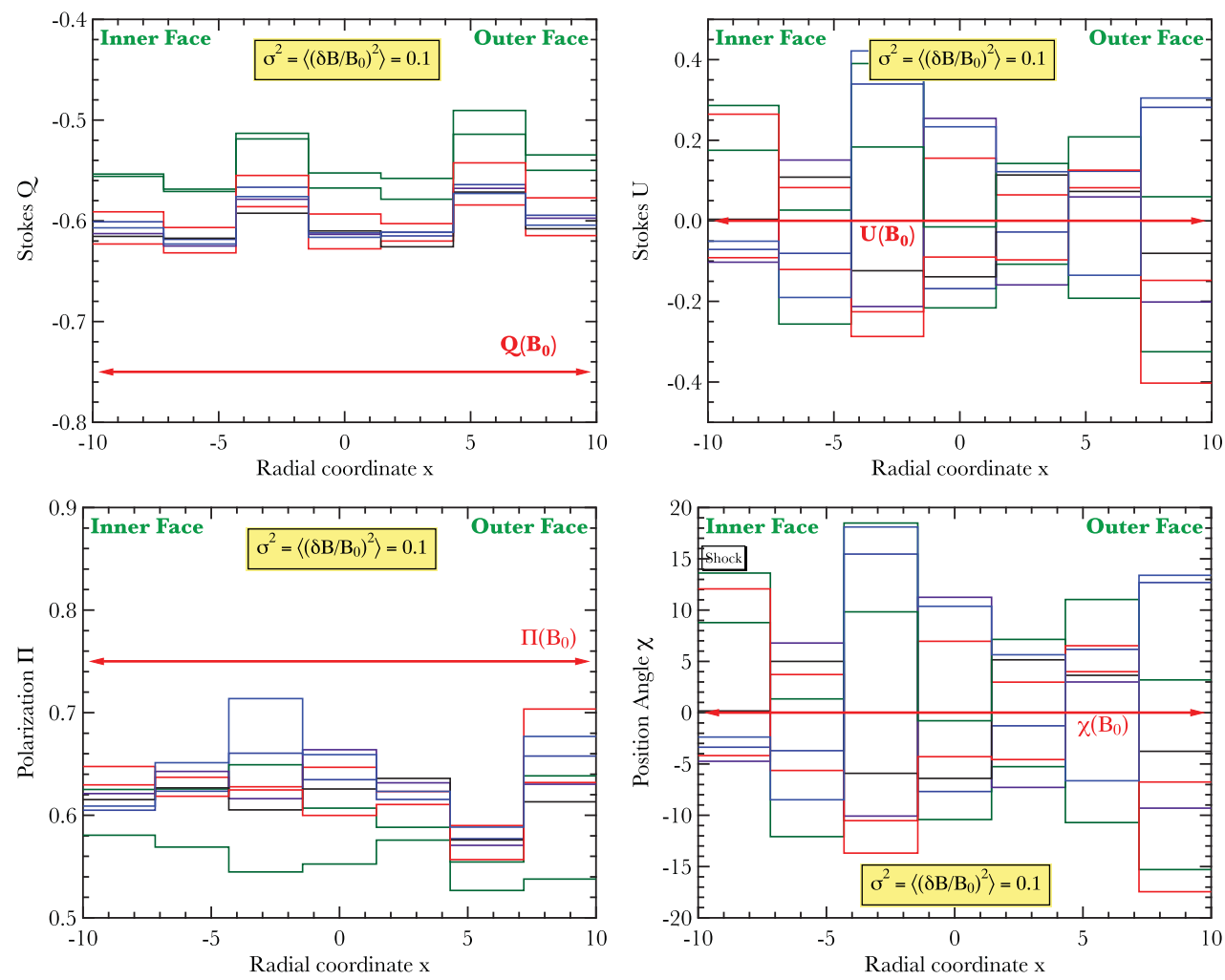

Figure 3. Synchrotron X-ray polarization quantities from power-law electrons as in Fig. 2, but here for a Kolmogorov spectrum with a higher variance of $\sigma^{2}=10^{-1}$ at the stirring scale. The enhanced depolarization and greater standard deviation in the position angle are evident.

the turbulence. We note that if IXPE measures polarization levels in excess of 30-40\%, theorists will have to revise the paradigm of turbulence generation in SNR shocks.

These brief insights elicit an exciting indication of the prospects for advances in our understanding of MHD turbulence in supernova remnant shells in the coming era of imaging X-ray polarimetry.

\section{References}

Bell, A. R. 2005, MNRAS, 358, 181

Bykov, A. M., Uvarov, Yu. A., Bloemen, J. B. G. M., et al. 2009, MNRAS, 399, 1119

Giacalone, J. \& Jokipii, J. R. 1999, Astrophys. J., 520, 204

Long, K. S., Reynolds, S. P., Raymond, J. C., et al. 2003, Astrophys J., 586, 1162

Lucek, S. G. \& Bell, A. R. 2000, MNRAS, 314, 65

Reynolds, S. P. \& Gilmore, D. M. 1993, Astronomical J, 106, 272

Reynoso, E. M., Hughes, J. P., \& Moffett, D. A. 2013, Astrophys. J., 145:104

Rybicki, G. B. \& Lightman, A. P. 1979 Radiative Processes in Astrophysics (Wiley, New York)

Soffitta, P., Barcons, X., Bellazzini, R., et al. 2013, Experimental Astronomy, 36, 523

Vink, J. \& Laming, J. M. 2003, Astrophys. J.,, 584, 758

Vladimirov, A., Ellison, D. C., \& Bykov, A. 2006, Astrophys. J., 652, 1246

Weisskopf, M. C., Baldini, L., Bellazini, R., et al. 2013, in Proc. SPIE, Vol. 8859, UV, X-Ray, and Gamma-Ray Space Instrumentation for Astronomy XVIII, 885908 